\title{
Simulation Study on Runway Threshold Stagger and Utilization Pattern of Closely Spaced Parallel Runways
}

\author{
Xiong $\mathrm{Li}^{1, \text { a }}$, Xiaoqing Chen ${ }^{2, \mathrm{~b}}$, Dongbin $\mathrm{Li}^{3, \mathrm{c}}$ and Dongxuan $\mathrm{Wei}^{4, \mathrm{~d}}$ \\ ${ }^{1}$ Planning and Design Institute, China Airport Construction Group Corporation of CAAC, China \\ ${ }^{2}$ Aviation Industry Development Research Center of China, China \\ ${ }^{3}$ Department of Air Traffic Management, Civil Aviation of Management Institute of China, China \\ ${ }^{4}$ Highway School, Chang'an University, China \\ alixiong_cacc@163.com, ${ }^{b} 18910528046 @ 189 . c n,{ }^{c}$ lidongbin@camic.cn, ddxwei@chd.edu.cn
}

Keywords: civil aviation; operation efficiency; computer simulation; parallel runways; Simmod Abstract. Closely spaced parallel runways(CSPRs) is the configuration mode frequently employed in planning and construction of the current airport runway. Simmod simulation software was operated here to construct the computer simulation models for different utilization patterns of runways, and made a well-organized analysis and quantitative evaluation of airport capacity and operational efficiency, given in the threshold staggering of CSPRs. Meanwhile, the effects of runway crossover and bypass-taxiing on operational efficiency of airport were also taken into account. The simulation result suggests: Abiding by IFR(instrument flight rules), a set of CSPRs are able to support 800 take-off and landing flights in peak day, while around 55 58 take-off and landing flights in peak hour. When the outside runway threshold is staggered toward the arriving aircraft and provided with the bypass taxiway, the take-off and landing efficiency of airport becomes the highest under the operational pattern(landing on the inside runway and taking off from the outside runway), about $3 \% \sim 7 \%$ higher than that under any other operational pattern.

\section{Introduction}

The continuous growth of social economy drives China air transport on the fast-growing track, during which China civil aviation has become the second largest air transport system in the world. The growing demand for air transport leads to the increasing scale of airport system in a response to the higher requirement. Currently, the majority of large airports in the said scale have constructed or planned several parallel runways to relieve the pressed demands on traffic volume. Therefore, it is of great significance to research the capacity of airport multi-runways system, particularly of parallel runways system, which will be used to instruct the construction of China airports in the future.

Three methods are usually employed to evaluate the capacity and operational efficiency of airport runways, i.e.: statistical data analysis, mathematical analysis and computer simulation. (1) Statistical data analysis is used to estimate the airport capacity based on the capacity envelope diagram drawn against the statistical flow data of existing airports, a model usually performed in the early stage of capacity analysis, but the disadvantage is that it can only evaluate the capacity of existing airport runways system ${ }^{[1,2]}$. (2) Mathematical analysis is used to obtain the capacity of runways by establishing the mathematical equation set of airport and airspace operational parameters through the appropriate hypothesis, which is mainly used for macro-level capacity evaluation ${ }^{[3-7]}$. (3) Computer simulation is an alternative in the most extensive application to evaluate the airport capacity and operational efficiency, which turns out the capacity level and efficiency index through the analysis of simulated operational data ${ }^{[8-11]}$. Simmod (Airport and Airspace Simulation Model) and TAAM (Total Airspace \& Airport Modeler) are the most popular simulation software for the evaluation of airport capacity $^{[12]}$.

However, the majority of present works as to airport capacity focuses on single runway ${ }^{[2,4]}$, the single operational mode of runways in the specific airport ${ }^{[5-10]}$ or the marco-level analysis ${ }^{[13]}$, while above study falls in short of intensive research on the capacity and operational efficiency of CSPRs, like considering different utilization patterns of airport runways, threshold staggering and utilization 
of crossover, bypass taxiways. In addition, CSPRs is one of the major patterns of runway configuration applied in current planning and construction of airport. In summary, this paper will intensively examine the utilization pattern and operational efficiency of CSPRs, operate Simmod to establish the computer simulation model, consider the effects of take-off and landing mode of different runways on airport capacity and operational efficiency in threshold even or staggering condition respectively, and make system analysis and quantitative evaluation, which may be theoretically referenced for the future planning and construction of CSPRs.

\section{Operational Mode of CSPRs}

Subject to the regulations of ICAO (International Civil Aviation Organization), parallel runways mean the uncrossed runways with parallel runway centerlines or the angle between the extending lines of centerlines is smaller than $15^{\circ}$, as in [14]. Closely spaced parallel runways(CSPRs) usually means the parallel runways with the centerline spacing between two runways no longer than $760 \mathrm{~m}$. In this configuration, terminal area is usually located at one side of runways rather than between two runways due to the small spacing between two parallel runways.

In thresholds even condition, the utilization pattern of CSPRs can be generally described as taking off from one side close to the terminal area(from the inside runway), and landing on the other side of runway(on the outside runway), as shown in Fig.1(a). Then landing flight is given two options: (1) After landing, crossing over the inside take-off runway to the terminal area; (2) After landing, bypass-taxiing to the terminal area. In this case, the runway capacity and operational efficiency have been examined under [13], which is not discussed here.

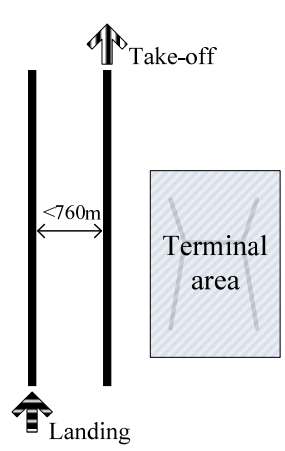

(a)

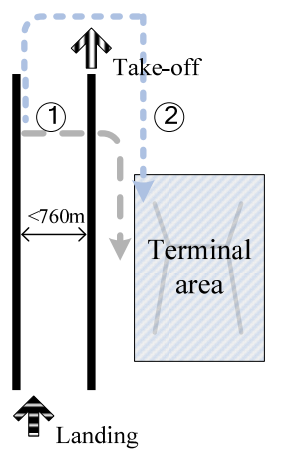

(b)

Fig. 1. Parallel runway thresholds even condition

In thresholds staggering condition, the layout of the representative runways and terminal area is analyzed, where thresholds staggering distance is smaller than $1,000 \mathrm{~m}$ and terminal area is located in the middle area of one side of runway. When the inside runway threshold is staggered toward the arriving aircraft, as shown in Fig.2(a), the utilization pattern of runways can be generally described as taking off from the inside runway, landing on the outside runway, and landing flights crossing over the inside take-off runway to the terminal area.

When the outside runway threshold is staggered toward the arriving aircraft, where the construction condition is applicable, the staggered space can be generally utilized to connect bypass taxiway with the outside runway. The utilization pattern of runways can be generally described as taking off from the inside runway, landing on the outside runway, as shown in Fig.2(b), and after landing, the flight is given two options (1) Crossing over the inside take-off runway, or (2) Bypass-taxiing to the terminal area. In addition, another alternative can be described as landing on the inside runway and taking off from the outside runway, as shown in Fig.2(c), all take-off flights bypass-taxi to the outside runway. 


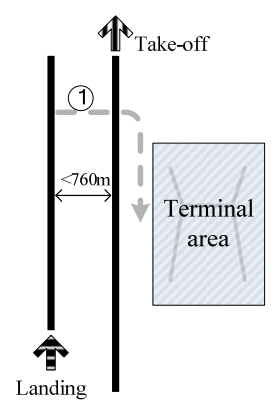

(a)

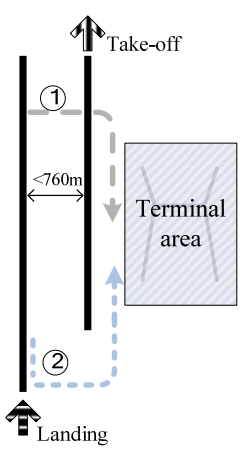

(b)

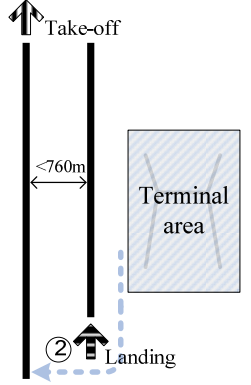

(c)

Fig. 2. Parallel runway thresholds staggering condition

\section{Control and Operation Separation}

Subject to ICAO regulations as to the operation of parallel runways, for all flights continuously taking off from or landing on CSPRs by IFR, the separation of take-off or landing should be executed like a single runway. Given no consideration of the airspace influence, when the flights continuously take off, the separation of take-off is controlled at 90 130 seconds according to the difference between front and follow aircraft type. For the flights continuously landing, the landing separation is mainly controlled by wake turbulence separation, as shown in Table 1 .

Table 1 Aircraft wake turbulence separation

\begin{tabular}{|c|c|c|c|c|}
\hline \multicolumn{5}{|c|}{ Aircraft Wake Turbulence Separation } \\
\hline Follow Front & $\mathbf{A 3 8 0}$ & Heavy & Middle & Light \\
\hline A380 & $8 \mathrm{~km}$ & $6 \mathrm{~km}$ & $6 \mathrm{~km}$ & $6 \mathrm{~km}$ \\
\hline Heavy & $12 \mathrm{~km}$ & $8 \mathrm{~km}$ & $6 \mathrm{~km}$ & $6 \mathrm{~km}$ \\
\hline Middle & $13 \mathrm{~km}$ & $10 \mathrm{~km}$ & $6 \mathrm{~km}$ & $6 \mathrm{~km}$ \\
\hline Light & $15 \mathrm{~km}$ & $12 \mathrm{~km}$ & $10 \mathrm{~km}$ & $6 \mathrm{~km}$ \\
\hline
\end{tabular}

In alternate operation of take-off and landing flights, when the take-off flight prepares to take-off roll aiming at the centerline, the distance between arriving aircraft and threshold must be greater than $5.5 \mathrm{~km}$. A certain of safe margin may be additionally left to the said control and operation separation in actual operation, generally in $15 \%$ or so.

\section{Simulation Modeling}

This paper operates Simmod Plus 7.6(simulation software) to make a simulation analysis of the operational efficiency in different operational patterns of CSPRs. Simmod, one dynamic and comprehensive airport simulation micro-software firstly proposed by FAA in 1978, through the continuous upgrading and perfection, has become one of the most applied airport and airspace simulation software ${ }^{[11,12]}$.

Given that this paper focally analyzes the capacity and efficiency of airport runways system, the simulation modeling will put aside the restrictive factors of airspace. In thresholds staggering condition, using one airport as the reference, three runway operational patterns are considered to establish the simulation model, corresponding to the operational pattern (a), (b) and (c) as shown in Fig.2. Pattern(a) - Inside runway staggered toward the arriving aircraft, taking off from the inside runway and landing on the outside runway; Pattern(b) -Inside runway staggered away from the arriving aircraft, taking off from the inside runway and landing on the outside runway; Pattern(c) Inside runway staggered away from the arriving aircraft, landing on the inside runway and taking off from the outside runway, as shown in Fig.3. Under Pattern (b) and (c), aircraft taxiing on the bypass taxiway will not interfere with the take-off, landing flights. 

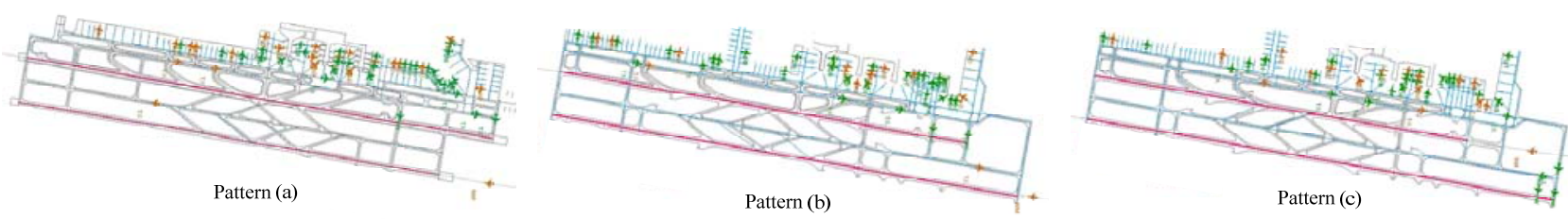

Fig. 3. Demonstration of simulation model

In the model, three sets of typical flight schedule are simulated and number of flights is set as shown in Table 2. Illustrating 760 flights per day, the waveform of daily take-off and landing flights is given in Fig.4. With reference to Chinese large airports, this paper assumes that the ratio of heavy and middle aircraft is $p_{h}=0.2, p_{m}=0.8$, respectively.

Table 2 Typical daily take-off and landing flights

\begin{tabular}{|c|c|c|c|c|}
\hline \multirow{2}{*}{$\begin{array}{c}\text { Annual Passenger Throughput } \\
\text { (in 10,000 person-time) }\end{array}$} & \multicolumn{3}{|c|}{ Flight Movements in Peak Day } & Movements in \\
Peak Hour
\end{tabular}

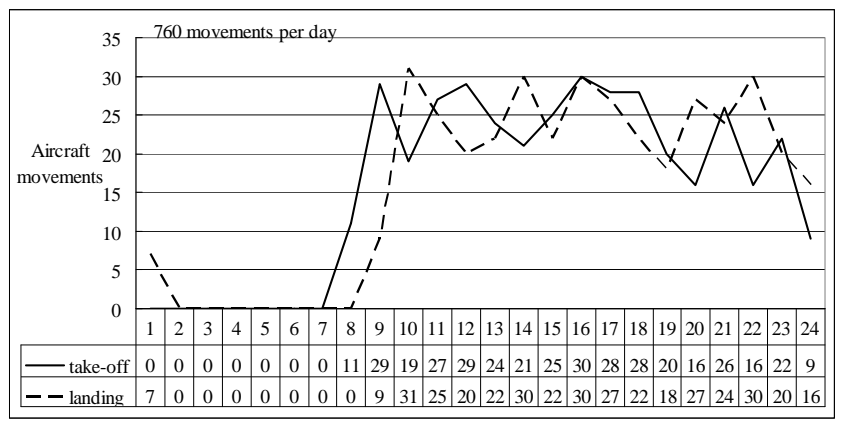

Fig. 4. Waveform of daily take-off and landing flights

\section{Analysis of Simulated Result}

Under three daily flights, after simulating the CSPRs operation under Pattern (a), (b) and (c), the statistical result of average delay time of departure, arrival flights is given in Table 3.

Table 3 Statistics of ground delay time

\begin{tabular}{|c|c|c|c|c|}
\hline Daily Flights & Category & Pattern(a) & Pattern(b) & Pattern(c) \\
\hline \multirow{2}{*}{596} & Departure & $2.58^{\prime}$ & $2.46^{\prime}$ & $2.48^{\prime}$ \\
\cline { 2 - 5 } & Arrival & $0.85^{\prime}$ & $0.70^{\prime}$ & $0.29^{\prime}$ \\
\hline \multirow{2}{*}{760} & Departure & $5.85^{\prime}$ & $4.94^{\prime}$ & $4.15^{\prime}$ \\
\cline { 2 - 5 } & Arrival & $0.95^{\prime}$ & $0.77^{\prime}$ & $0.30^{\prime}$ \\
\hline \multirow{2}{*}{880} & Departure & $10.53^{\prime}$ & $9.70^{\prime}$ & $8.63^{\prime}$ \\
\cline { 2 - 5 } & Arrival & $1.07^{\prime}$ & $0.85^{\prime}$ & $0.37^{\prime}$ \\
\hline
\end{tabular}

Under Pattern (a), ground delay time is the longest, and the average ground delay time of departure flights increases around 10.6\%, 22.3\% compared to that under Pattern (b) and Pattern (c) respectively. The longer ground delay time of departure flights can be mainly attributed to: inside runway staggered toward the arriving aircraft, planning bypass taxiway may substantially increase the taxiing distance of flight, crossing over the runway provided with no bypass taxiway will lead to the reduced release efficiency of departure flights.

When the number of daily flights is bigger, Pattern(c) further decreases the ground delay time of departure flights by around 11 16\% compared to that under Pattern(b), which can be mainly attributed to: (1) Under Pattern(c), departure flights take off from the outside runway, the bypass 
taxiway is utilized to provide the sufficient queuing space and diminish the interference with the flights operating in the terminal area; (2) The arrival or departure flights don't need to cross over the runway, improving the operational efficiency. Besides, under different operational patterns, the ground delay time of arrival flights is shorter (around 1 minute).

With reference to the simulation experience of large airport, using the average 6 minutes ground delay of departure flights as the delay standard to measure the normal operation of airport[15]. The airport take-off and landing capacity under Pattern(a), (b) and (c) is 765, 795 and 815 flights per day, as shown in Fig.5.

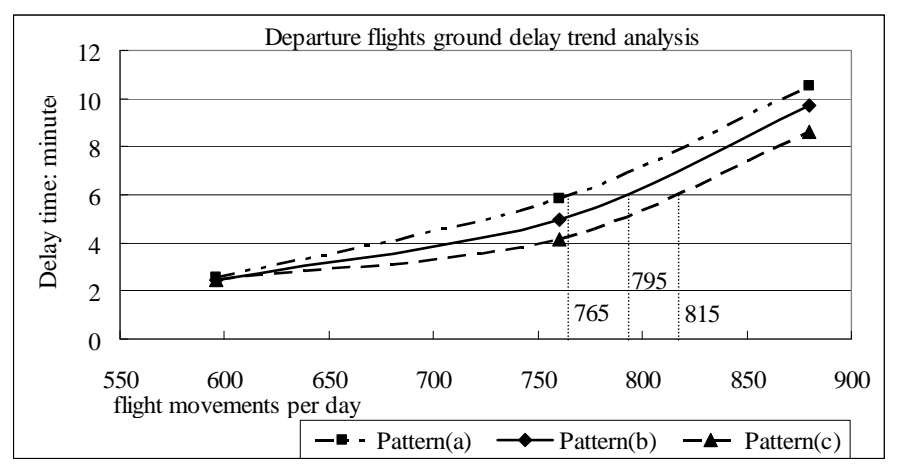

Fig. 5. Ground delay trend of departure flights

Based on Pattern(b), the ground operational efficiency is further analyzed, provided that all landing flights on the outside runway travel to the terminal area via bypass taxiway (crossover taxiway is disabled). When the number of daily flights is 760 , the average ground delay time of departure, arrival flights is 3.29, 0.91 minutes respectively, 1.65 minutes shorter than that of departure flights, and 0.14 minutes longer than that of arrival flights provided that the crossover runway is enabled. Because all landing flights utilize the bypass taxiway, the average taxiing time of landing flights sharply increases to the value about 58\% longer than that when the crossover runway is enabled.

Under different operational patterns, smaller difference is identified between take-off and landing flights in peak hour, around 55 58 flights/hour. Illustrating Pattern(b), when the number of daily flights is 760, the take-off and landing flights in 24 hours(peak day) are given in Fig.6.

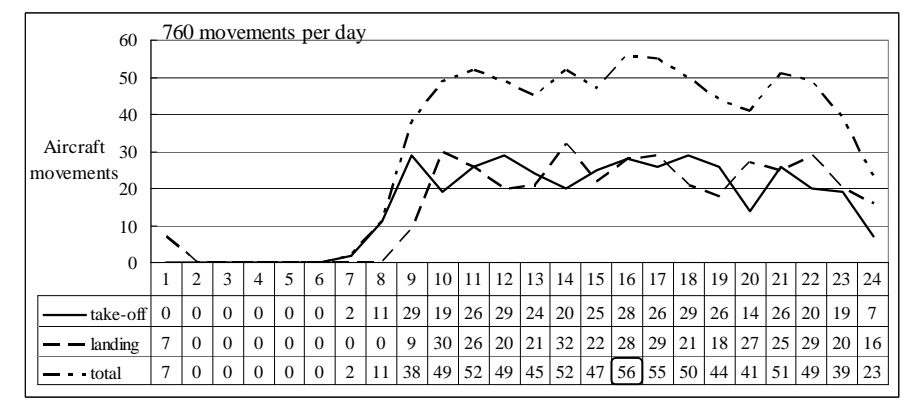

Fig. 6. Statistics of daily take-off and landing flights

\section{Conclusions}

This paper operates Simmod simulation software to establish the computerized simulation model for different utilization patterns of runways, and make systematic analysis and quantitative evaluation of airport capacity and operational efficiency in CSPRs thresholds staggering condition, with the following conclusions:

(1) By the prevailing ICAO's IFR, a set of CSPRs are able to support 800 take-off and landing flights in peak day, while around 55 58 take-off and landing flights in peak hour.

(2) For CSPRs, when the outside runway threshold is staggered toward the arriving aircraft, the airport operational efficiency is the highest under Pattern(c) (landing on the inside runway and taking 
off from the outside runway), provided that the bypass taxiway is applicable. The efficiency of take-off and landing can increase around 3\% 7\% compared to that under other operational patterns.

(3) When the flights land on the inside, or outside runway, the bypass taxiway can be utilized to appropriately reduce the flight delay, but it may result in the sharp increase of taxiing time of some flights. In the course of actual operation, flights should be rationally allocated the utilization of crossover and bypass taxiway.

Due to the length limit, this paper only does the simulation study on the representative configurations and operational patterns of CSPRs, and attempts to provide the necessary theoretical justifications for the planning and construction of airport runways in the future. For the analysis of atypical CSPRs configurations, more specific study remains to be done by taking the overall airfield layout into account.

\section{Acknowledgements}

This work was financially supported by the National Key Technologies R\&D Program (2014BAJ04B02).

\section{References}

[1] E.P. Gilbo: IEEE Transactions on Control Systems Technology, Vol. 1 (1993), p. 144-154.

[2] Minghua Hu, Song Liu, Langen Su: Journal of Data Acquisition \& Processing, Vol. 15 (2000), p. 74-77. In Chinese

[3] R.D. Neufville, A.R. Odoni: Airport Systems Planning, Designs, and Management ( McGrawHill, New York 2002).

[4] Jing Yu, Yun Pu: Journal of Traffic and Transportation Engineering, Vol. 2 (2002), p. 99-102. In Chinese

[5] Yong Chen, Yihua Cao, Yong Zhou: Flight Dynamics, Vol. 23 (2005), p. 86-89. In Chinese

[6] Haiqi Guo, Jinfu Zhu: Journal of Traffic and Transportation Engineering, Vol. 8 (2008), p. 68-72. In Chinese

[7] Xin Chen: Research on Capacity Evaluation and Optimization Methods at Airport Airside, Nanjing University of Aeronautics \& Astronautics (2007). In Chinese

[8] Chao. Wang, Xinyue Zhang, Xiaohao Xu: Simulation study on airfield system capacity analysis using SIMMOD, in: International Symposium on Computational Intelligence and Design, Wuhan(2008), p. 87-90.

[9] Wei Gao, Siyuan Jiang: Simulation study on closely spaced parallel runway analysis using SIMMOD plus, in: Proceedings of the 2010 International Conference on Intelligent Computation Technology and Automation, Los Alamitos, CA(2010), p. 344-347.

[10] Wei Gao, Chaowei Huang: Journal of Civil Aviation Univ. of China, Vol. 28(2010), p. 1-4. In Chinese

[11] Haiyang Yu, Huaqing Hu, Jinjin Yao: Air Traffic Management, Vol. 2(2009), p. 21-25. In Chinese.

[12] A.S. Huang, D. Schleicher, G. Hunter: Future flight demand generation tool, in: AIAA 4th Aviation Technology, Integration and Operations (ATIO) Forum, Chicago, IL(2004), p. 6400.

[13] Xiong Li, Dongbin Li, Dongxuan Wei: Journal of Computer Applications, Vol. 32(2012), p. 2648-2651. In Chinese

[14] Kai Li: Study of Approach Procedure to Closely Spaced Parallel Runways, Nanjing University of Aeronautics \& Astronautics (2005). In Chinese

[15] Federal Aviation Administration: Airport Master Plans, AC No. 150/5070-6B (2015), p. 51. 